\title{
Energy Efficient Device to Device Communication by Resource Efficiency Optimization
}

DOI:

10.1109/VTCSpring.2017.8108255

\section{Document Version}

Accepted author manuscript

Link to publication record in Manchester Research Explorer

\section{Citation for published version (APA):}

Idris, F., Tang, J., \& So, K. C. (2017). Energy Efficient Device to Device Communication by Resource Efficiency Optimization. In IEEE Vehicular Technology Conference - Spring 2017 IEEE.

https://doi.org/10.1109/VTCSpring.2017.8108255

\section{Published in:}

IEEE Vehicular Technology Conference - Spring 2017

\section{Citing this paper}

Please note that where the full-text provided on Manchester Research Explorer is the Author Accepted Manuscript or Proof version this may differ from the final Published version. If citing, it is advised that you check and use the publisher's definitive version.

\section{General rights}

Copyright and moral rights for the publications made accessible in the Research Explorer are retained by the authors and/or other copyright owners and it is a condition of accessing publications that users recognise and abide by the legal requirements associated with these rights.

\section{Takedown policy}

If you believe that this document breaches copyright please refer to the University of Manchester's Takedown Procedures [http://man.ac.uk/04Y6Bo] or contact uml.scholarlycommunications@manchester.ac.uk providing relevant details, so we can investigate your claim.

\section{OPEN ACCESS}




\title{
Energy Efficient Device to Device Communication by Resource Efficiency Optimization
}

\author{
Fakrulradzi Idris*, Jie Tang ${ }^{\dagger}$, and Daniel K. C. So* \\ ${ }^{*}$ School of Electrical and Electronic Engineering, University of Manchester, United Kingdom \\ ${ }^{\dagger}$ School of Electronics and Information Engineering, South China University of Technology, Guangzhou, China \\ Email: fakrulradzi.idris@postgrad.manchester.ac.uk, eejtang@scut.edu.cn, d.so@manchester.ac.uk
}

\begin{abstract}
Device-to-Device (D2D) communication is one of the technologies for next generation communication system. Unlike traditional cellular network, D2D allows proximity users to communicate directly with each other without routing the data through a base station. In this paper, we propose a resource allocation scheme for energy efficiency (EE) optimization in cellular network with overlaying D2D communication. The objective of this work is to maximize the overall $E E$ of the network while satisfying the rate and power constraints for all users. We decompose the main problem into two subproblems; resource efficiency (RE) optimization for cellular user in the first stage and EE optimization for D2D pair in the second stage. The RE optimization problem is solved using the bisection method while Dinkelbach and interior point method are implemented to solve the EE optimization problem. Simulation results demonstrate that the proposed scheme outperforms the cellular mode and dedicated mode of communication and the performance is close to the global optimal solution.
\end{abstract}

Index Terms-Device-to-Device (D2D) communication; resource efficiency; energy efficiency.

\section{INTRODUCTION}

Device-to-Device (D2D) communication [1] enables user equipment (UE) to communicate directly with each other, without routing the data paths through a central infrastructure such as evolved Node B (eNB) or access point. Bluetooth and WiFi are two examples of traditional D2D communication as devices in the close range can operate in ad-hoc manner for file transfer or information sharing. Both Bluetooth and WiFi are operating in unlicensed ISM band. However, D2D communication in cellular network depends on licensed frequency and shares the spectrum with other cellular users. Introducing D2D communication in a cellular network will improve the overall data rate, energy consumption and spectrum utilization, while providing new services [2]. Therefore, D2D communication is also regarded as one of the key technology components for $5 \mathrm{G}$ wireless networks [3], [4].

System performances in terms of coverage and spectral efficiency have always been the focus of the network designers. Coverage performance can be obtained by studying the distribution of signal to the interference and noise ratio (SINR) of users while spectral efficiency (SE) provides a measure of how many bits of information can be transferred to the served users for a given bandwidth. Another metric that has attracted much attention is the energy efficiency (EE) of the network.
Energy efficient D2D communications for a single cellular user and a D2D pair have been investigated in [5]-[7]. In particular, three different uplink scenarios which are nonorthogonal sharing mode (also known as reusing mode), orthogonal sharing mode (or dedicated mode) and cellular mode are studied in [5], [6]. In reusing mode, resources are reused by cellular and D2D users while in dedicated mode, exclusive resources are assigned for cellular and D2D users. In cellular mode, D2D users communicate with each other via eNB same like the traditional cellular network. To maximize the D2D pair's EE while considering the data rate requirements of all users, a distributed algorithm is proposed in [7]. In that work, three different regions for the D2D's circuit power consumption are defined and the optimal power control for each region is derived. Nevertheless, the power amplifier efficiency has been ignored in all these work.

Recently, a new metric called resource efficiency (RE) has been introduced in [8] to study the tradeoff between EE and SE. By using the RE approach, significant amount of bandwidth can be saved with a slight increase in power consumption. Similarly, it was also found that significant amount of power can be reduced by slight bandwidth expansion. Therefore, RE is capable to optimize both EE and SE simultaneously by balancing the power consumption and occupied bandwidth. The main idea of this work is to maximize the $\mathrm{RE}$ of cellular user to provide bandwidth saving in macro cell network. The remaining bandwidth can be used to optimize the EE of D2D user.

In this paper, we study the joint RE and EE problem that aims to maximize the system $\mathrm{EE}$ and guarantee the data rate requirements as well as power constraints for both cellular user and D2D pair. Specifically, we develop a scheme to solve the optimization problem in two stages. In the first stage, the RE problem for cellular user is formulated and solved using the bisection method. Then, the EE optimization problem for D2D pair is solved in the second stage. To solve the EE problem, Dinkelbach algorithm is adopted to transform the fractional problem into subtractive form. Interior point method is then used to solve the transformed EE problem efficiently.

The remaining of the paper is organized as follows. The system model and problem formulation are described in Section II. In Section III, the proposed RE and EE optimization are considered and related algorithms are developed. Then we present simulation results in Section IV. Finally, conclusions 
are drawn in Section V.

\section{System Model And Problem Formulation}

\section{A. System Model}

A single isolated cell is considered and illustrated in Fig. 1. The basic D2D communication scenario consists of one cellular user (CUE), UE1 and one D2D pair (UE2 and UE3) in which UE2 is the D2D transmitter. The eNB is located at the center of the cell. The D2D pair (DUE) operates in an overlay manner and does not share the uplink radio resources with the CUE. Hence, there is no interference from the D2D transmitter towards the eNB. In this work, the CUE has the priority to utilize available resources to communicate with the eNB.

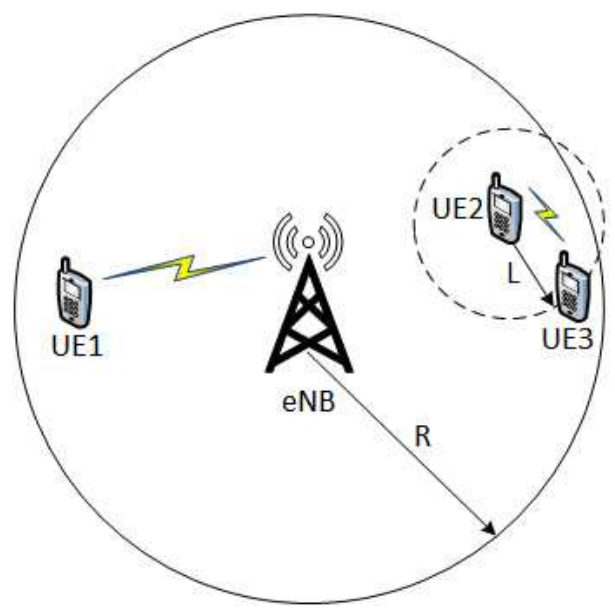

Fig. 1. System model of D2D communications

Let $h_{c B}$ and $h_{d d}$ be the channel gain of the link between CUE and eNB and D2D link respectively. The SINR of the cellular link can be expressed as

$$
\gamma_{c}=\frac{P_{c} h_{c B}}{W_{c} N_{0}}
$$

where $P_{c}$ represents the transmit power of the CUE, $W_{c}$ denotes the allocated spectrum resources for CUE, and $N_{0}$ is the noise power spectral density. Similarly, the SINR of DUE can be written as

$$
\gamma_{d}=\frac{P_{d} h_{d d}}{W_{d} N_{0}}
$$

where $P_{d}$ denotes the transmit power of the D2D transmitter and the allocated spectrum resources for D2D is given by $W_{d}$.

The data rate of CUE, $R_{c}$ and data rate of DUE, $R_{d}$ can be calculated as

$$
\begin{aligned}
& R_{c}=W_{c} \log _{2}\left(1+\gamma_{c}\right) \\
& R_{d}=W_{d} \log _{2}\left(1+\gamma_{d}\right)
\end{aligned}
$$

The overall power consumption for the CUE and D2D links consist of transmit power and circuit power which can be modeled as

$$
P=\varepsilon P_{t}+P_{c i r}
$$

where $\varepsilon$ is the inverse of power amplifier efficiency, $P_{t}$ is the transmit power and $P_{c i r}$ is the circuit power.

\section{B. Problem Formulation}

In this paper, we consider two optimization problems with the following constraints. First, it is required to achieve the minimum rate for each cellular link and D2D link

$$
\begin{aligned}
& R_{c} \geq R_{c}^{\text {min }} \\
& R_{d} \geq R_{d}^{\text {min }}
\end{aligned}
$$

where $R_{c}^{\text {min }}$ and $R_{d}^{\text {min }}$ denote the minimum data rates for the CUE and the DUE, respectively. Second, the power constraints of individual links are given as

$$
\begin{aligned}
& P_{c} \leq P_{c}^{\max } \\
& P_{d} \leq P_{d}^{\max }
\end{aligned}
$$

where $P_{c}^{\max }$ and $P_{c}^{\max }$ are the maximum transmission power of the CUE and the DUE, respectively.

The first problem aims to maximize the RE for CUE. Introduced in [8], RE is defined as

$$
R E=\frac{R}{P}\left(1+\beta \frac{\eta_{p}}{\eta_{w}}\right)
$$

where $R$ is the overall throughput and $P$ denotes the overall power consumption as in (5). $\beta$ is used as a weighted factor to control the balance of EE and SE. Power utilization and bandwidth utilization are denoted by $\eta_{p}$ and $\eta_{w}$ respectively and given by

$$
\begin{aligned}
\eta_{p} & =\frac{P}{P_{t o t}} \\
\eta_{w} & =\frac{W}{W_{t o t}}
\end{aligned}
$$

where $P_{t o t}=\varepsilon P_{c}^{\max }+P_{c i r}, W$ is the occupied bandwidth, and $W_{\text {tot }}$ is the total bandwidth.

Therefore, the RE optimization for CUE can be formulated as

$$
\begin{aligned}
& \max _{W_{c}, P_{c}} R E_{C}=\frac{R_{c}}{P}\left(1+\beta \frac{\eta p}{\eta w}\right) \\
& \text { s.t. } \quad(6),(8) \text { and } W_{c} \leq W_{\text {tot }}
\end{aligned}
$$

The second problem maximizes the EE of the DUE which can be expressed as

$$
\begin{gathered}
\max _{P_{d}} E E_{D}=\frac{R_{d}}{\varepsilon P_{d}+P_{\text {cir }}} \\
\text { s.t } \quad(7),(9)
\end{gathered}
$$

It must be noted that there is no bandwidth constraint for this second problem as EE optimization always fully utilize 
the available bandwidth. Therefore, the bandwidth for DUE is always $W_{t o t}-W_{c}$.

We present algorithms to solve these problems in the following section.

\section{PRoposed RE AND EE OptimizATION}

\section{A. Resource Efficiency Optimization for Cellular user}

$\mathrm{RE}$ is introduced to optimize both EE and SE simultaneously. Generally, the CUE has the priority to transmit signals without being interfered by other users. In this stage, RE maximization for cellular user is performed to obtain the optimal transmission power and bandwidth allocation required to guarantee the quality of service (QoS).

The optimization problem (13) is difficult to be solved directly due to the non-convex fractional structure of the objective. As RE is a two variables problem, we can use a third dimension (3D) plot to study the relationship between the objective function and the optimization variables. Fig. 2 shows the surface plots of the RE objective function with respect to power and bandwidth constraint sets for different values of $\beta$.

The surface plots show that RE is a quasiconcave function. Specifically, we can verify that RE is quasiconcave in the transmit power for a given value of $\beta$ and $W_{c}$. Furthermore, the gradient of RE vanishes at the optimal point. Therefore, the bisection method [9] is used to solve the quasiconcave problem.

It is also interesting to note that when $\beta=0$, problem (13) simply becomes an EE optimization problem which requires the most of the bandwidth. However, for a larger value of $\beta$ such as $\beta=10$, the maximum $\mathrm{RE}$ can be achieved while requiring much lower bandwidth.

To solve the RE problem with respect to transmit power using bisection method, the gradient equation of RE is required. For a fix value of bandwidth, the gradient of $\mathrm{RE}$ with respect to power can be derived as

$$
\frac{d R E\left(P_{c}\right)}{d\left(P_{c}\right)}=\frac{(A / B)-C}{\left(\varepsilon P_{c}+P_{c i r}\right)^{2} \ln (2)}
$$

where

$$
\begin{aligned}
A= & H_{c B}\left(\varepsilon P_{c}+P_{c i r}\right)\left(\left(P_{c i r}+\varepsilon P_{c}^{\max }\right) W_{c}\right. \\
& \left.+\beta\left(\varepsilon P_{c}+P_{c i r}\right) W_{t o t}\right), \\
B= & \left(P_{c i r}+\varepsilon P_{c}^{\max }\right)\left(H_{c B} P_{c}+N_{0} W_{c}\right), \\
C= & \varepsilon W_{c} \ln \left(1+\frac{H_{c B} P_{c}}{N_{0} W_{c}}\right),
\end{aligned}
$$

Table I summarizes the algorithm to solve the RE optimization problem.

In each iteration, the value of $\mathrm{RE}$ is calculated. The maximum value of $\mathrm{RE}$ which satisfies the rate constraint is selected and the corresponding $W_{c}^{*}$ and $P_{c}^{*}$ can be obtained. In Algorithm 1, the transmission power constraint is considered in the interval $\left[z_{a}, z_{b}\right]$. The complexity of bisection method is given as $\mathcal{O}\left(\log _{2} n\right)$ where $n=\frac{z_{b}-z_{a}}{\epsilon}$. To solve the overall RE problem, the number of iteration required is $K$ times, where $K=\left(W_{\text {tot }} / W_{\text {step }}\right)$.

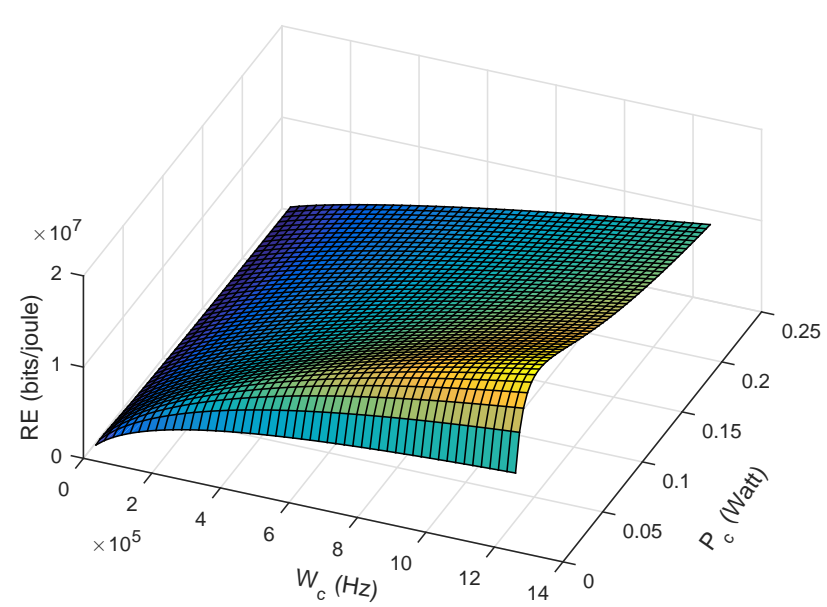

(a)

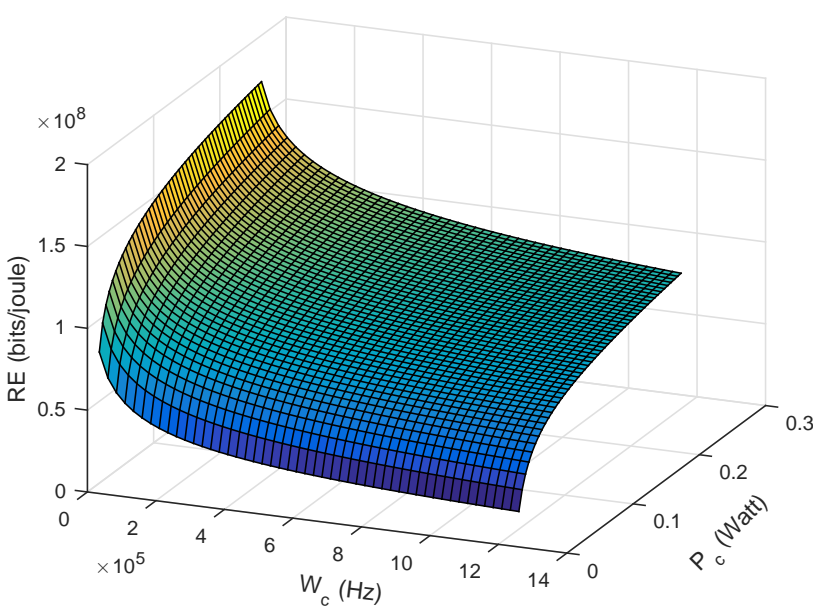

(b)

Fig. 2. Surface plots of $\operatorname{RE}(a) \beta=0,(b) \beta=10$

\section{B. Energy Efficiency Optimization for D2D pair}

In the second stage, the D2D pair will use all remaining bandwidth to maximize its EE. The objective function in (14) is non convex due to fractional form. According to [10], this function can be transformed into a convex optimization function by using nonlinear fractional programming in [11]. Specifically, (14) can be transformed into a new parameterized concave function with a parameter $q$, which can be represented as

$$
\begin{gathered}
\max _{P_{d}} \quad \eta_{E E}=R_{d}-q\left(\varepsilon P_{d}+P_{c i r}\right) \\
\text { s.t } \quad(7),(9)
\end{gathered}
$$

Based on the nonlinear fractional programming theory, the maximum $\mathrm{EE} q^{*}=\frac{R_{d}}{\varepsilon P_{d}^{*}+P_{\text {cir }}}$ is achieved if and only if $\max _{P_{d}} \quad R_{d}-q^{*}\left(\varepsilon P_{d}+P_{c i r}\right)=R_{d}-q^{*}\left(\varepsilon P_{d}^{*}+P_{c i r}\right)=$ 0 . This indicates that an equivalent optimization problem 
TABLE I

ALGORITHM FOR RE OPTIMIZATION

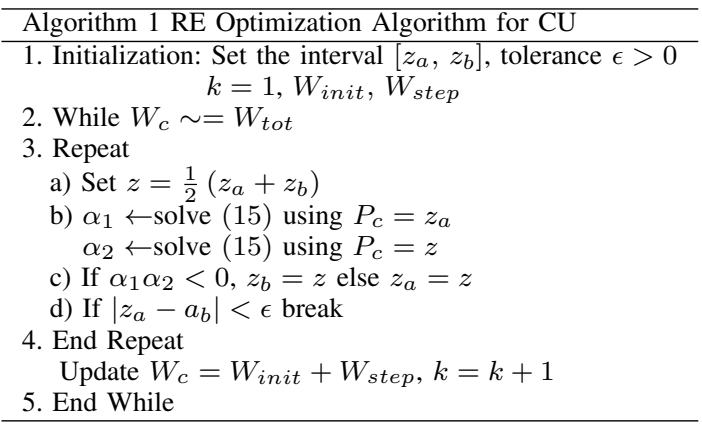

exists with an objective function in the subtractive form. Furthermore, the problem now becomes a convex optimization problem due to the concavity of the objective function and the constraints are convex [6]. Therefore, the Dinkelbach method [11] and interior point method can be used to obtain the optimal solution to the problem. The energy efficient algorithm for problem (16) is presented in Table II.

TABLE II

ALGORITHM FOR EE OPTIMIZATION

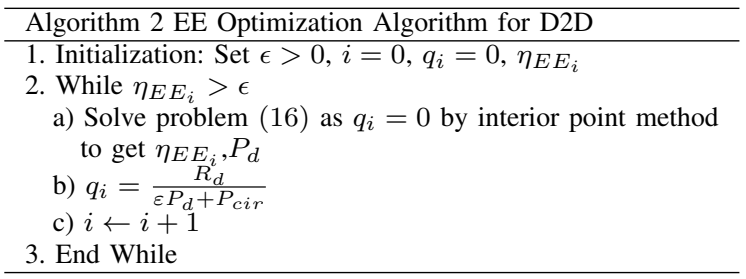

\section{Simulation Results}

In this section, we evaluate the performance of the proposed optimal RE and EE optimization scheme. In the simulation, both cellular user and D2D transmitter are uniformly distributed in a cell. D2D receiver is randomly located with a distance of $L$ from D2D transmitter. The channel gain between transmitter $i$ and receiver $j$ is calculated as as $h_{i, j}=c_{0} \varsigma_{i, j} \kappa_{i, j} d_{i, j}^{-\alpha}$, where $c_{0}$ is the pathloss coefficient, $\varsigma_{i, j}$ is the shadowing, $\kappa_{i, j}$ is the squared magnitude of the Rayleigh fading, $d_{i, j}$ is the distance between transmitter and receiver, and $\alpha$ is the pathloss exponent. The channel model parameters include pathloss coefficient of $-20 \mathrm{~dB}$, unit mean for the Rayleigh fading process, a pathloss exponent of 4 , and a standard deviation of $8 \mathrm{~dB}$ for the log-normal shadowing. Table III summarizes the main simulation parameters.

The impact of the weighted parameter $\beta$ to EE and SE is depicted in Fig. 3. It shows that the EE is non-increasing with $\beta$, whereas SE is non-decreasing with $\beta$. At a certain point, the value of RE's weighted factor could provide the tradeoff between RE and $\mathrm{EE}$ for the cellular user in the macro cell network. The cross point between EE and SE occurs when $\beta=2.25$. Fig. 4 compares the plot of the proposed bandwidth and power allocation schemes with the global optimal solution.
TABLE III

SIMULATION PARAMETERS

\begin{tabular}{|c|c|}
\hline Parameters & Value \\
\hline \hline Spectrum bandwidth $\left(W_{\text {tot }}\right)$ & $1.25 \mathrm{MHz}$ \\
\hline Cell radius $(R)$ & $500 \mathrm{~m}$ \\
\hline D2D pair distance $(L)$ & $20,40, \ldots, 120 \mathrm{~m}$ \\
\hline Maximum transmit power of CU $\left(P_{c}^{\text {max }}\right)$ & $250 \mathrm{~mW}$ \\
\hline Maximum transmit power of D2D $\left(P_{d}^{\text {max }}\right)$ & $250 \mathrm{~mW}$ \\
\hline Minimum rate of CU and D2D $\left(R_{c}^{\text {min }}, R_{d}^{\text {min }}\right)$ & {$[0,5] \mathrm{Mbit}$} \\
\hline Circuit power $\left(P_{c i r}\right)$ & $100 \mathrm{~mW}$ \\
\hline Noise power spectral density $\left(N_{0}\right)$ & $-174 \mathrm{dBm} / \mathrm{Hz}$ \\
\hline
\end{tabular}

From the figure, it can be seen that the proposed schemes allocate the bandwidth and power effectively and are similar to the global optimal solution. More importantly, this shows the relationship of RE optimization to power and bandwidth, whereby when $\beta=0$, the optimization is focused on EE and the bandwidth is fully utilized while the power is minimized. On the other hand when $\beta$ is high, the focus is on SE and results are reversed.

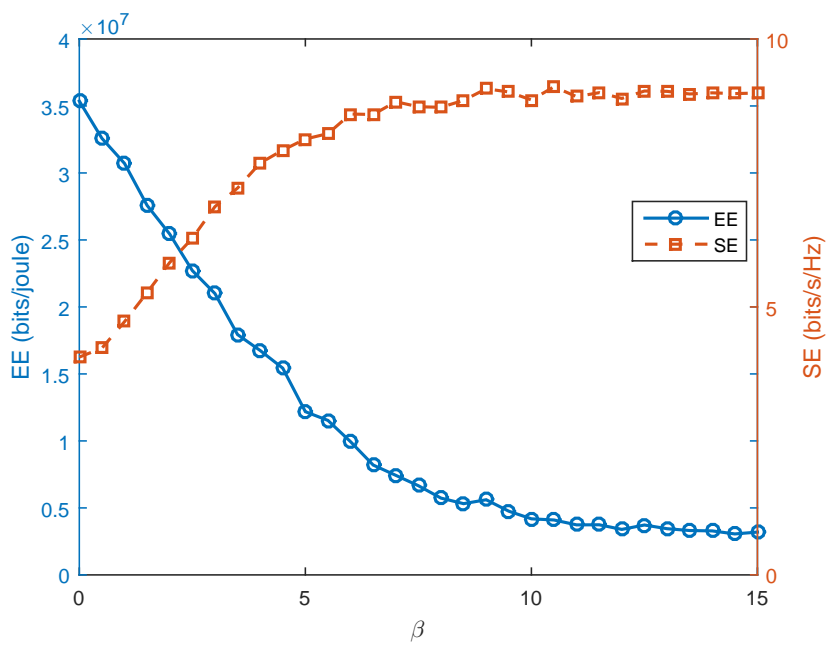

Fig. 3. Impact of weighted parameter to EE and SE

Fig. 5 shows the individual EE for CUE and DUE versus the D2D separation for $\beta=1$. The close proximity between D2D pair enables D2D communication to achieve higher EE than CUE. However, as the distance between D2D pair increases, more transmission power is required to overcome the deteriorating channel condition. As a result, the EE for D2D pair decreases. It can also be observed that the EE does not change for the CUE as the D2D users are communicating in an overlay manner which cause no interference to the CUE.

Fig. 6 compares the total EE performance of the proposed scheme with the high complexity global optimal solution. In addition, it is also compared to the dedicated mode with the bandwidth and power for the cellular and D2D pair being optimized simultaneously, and the cellular mode where all users are routed through the eNB. From the figure, the performance of the propose scheme is close to the global optimal solution but with much lower complexity due to the 


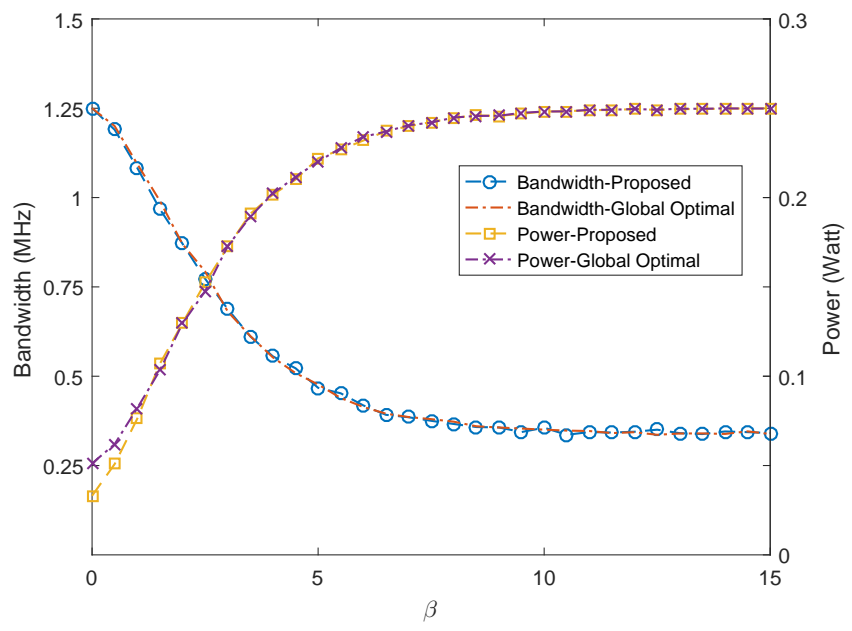

Fig. 4. Bandwidth and power allocation

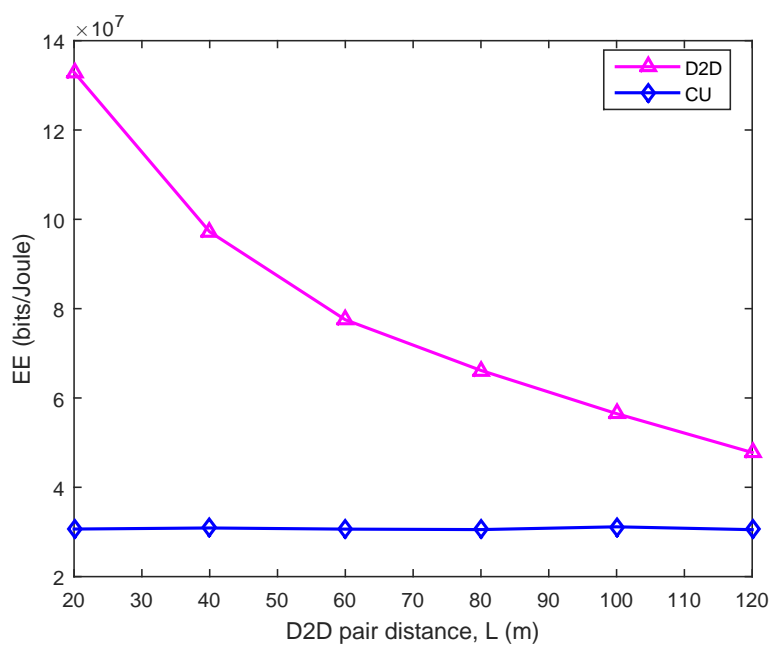

Fig. 5. EE versus D2D pair distance

two stage optimization. Moreover, the proposed method is better than the dedicated and cellular mode. Therefore, the proposed scheme can provide an energy efficient solution for the D2D enabled cellular system.

\section{CONCLUSIONS}

In this paper, we propose a scheme which combines the resource efficiency and energy efficiency for cellular network overlaid by D2D communication. In the first stage, resource efficiency optimization is formulated and solved by applying an iterative algorithm with bisection method. Using a weighted parameter to control the available resources for cellular user, the D2D pair can use the remaining bandwidth to maximize its energy efficiency. In the second stage, Dinkelbach and interior point methods were exploited to obtain the optimal power allocation for D2D pair. Simulation results showed that the proposed scheme offers higher overall energy efficiency compared to cellular and dedicated mode of transmission. In

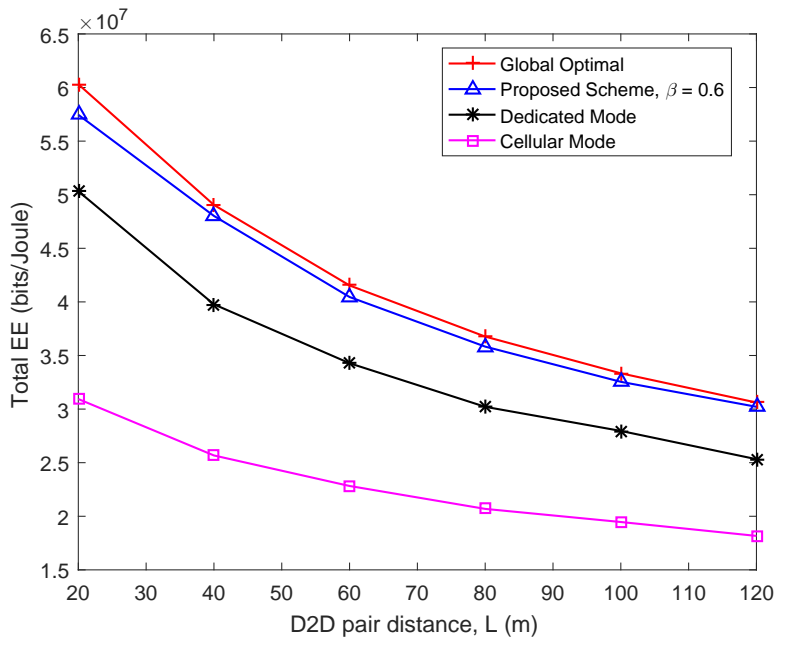

Fig. 6. Total EE versus D2D pair distance

addition, the proposed scheme perform close to the global optimal solution but with lower complexity.

\section{ACKNOWLEDGMENT}

The first author would like to thank Ministry of Higher Education Malaysia, and Universiti Teknikal Malaysia Melaka (UTeM) for funding the research.

\section{REFERENCES}

[1] K. Doppler, M. Rinne, C. Wijting, C. Ribeiro, and K. Hugl, "Deviceto-device communication as an underlay to lte-advanced networks," Communications Magazine, IEEE, vol. 47, no. 12, pp. 42-49, Dec 2009.

[2] X. Lin, J. Andrews, A. Ghosh, and R. Ratasuk, "An overview of 3gpp device-to-device proximity services," Communications Magazine, IEEE, vol. 52, no. 4, pp. 40-48, April 2014.

[3] W. H. Chin, Z. Fan, and R. Haines, "Emerging technologies and research challenges for 5G wireless networks," Wireless Communications, IEEE, vol. 21, no. 2, pp. 106-112, April 2014.

[4] L. Song, D. Niyato, Z. Han, and E. Hossain, Wireless Device-to-Device Communications and Networks. Cambridge, UK: Cambridge University Press, 2015.

[5] X. Qiu, L. Xuewen, K. Dong, and S. Zhu, "Energy efficiency analysis in device-to-device communication underlaying cellular networks," in Consumer Communications and Networking Conference (CCNC), 2013 IEEE, Jan 2013, pp. 625-630.

[6] D. Feng, G. Yu, C. Xiong, Y. Yuan-Wu, G. Y. Li, G. Feng, and S. Li, "Mode switching for energy-efficient device-to-device communications in cellular networks," IEEE Transactions on Wireless Communications, vol. 14, no. 12, pp. 6993-7003, Dec 2015.

[7] Y. Wu, J. Wang, L. Qian, and R. Schober, "Optimal power control for energy efficient $\mathrm{d} 2 \mathrm{~d}$ communication and its distributed implementation," Communications Letters, IEEE, vol. 19, no. 5, pp. 815-818, May 2015.

[8] J. Tang, D. So, E. Alsusa, and K. Hamdi, "Resource efficiency: A new paradigm on energy efficiency and spectral efficiency tradeoff," Wireless Communications, IEEE Transactions on, vol. 13, no. 8, pp. 4656-4669, Aug 2014.

[9] S. Boyd and L. Vandenberghe, Convex Optimization. Cambridge, UK Cambridge University Press, 2004.

[10] Z. Zhou, M. Dong, K. Ota, J. Wu, and T. Sato, "Distributed interferenceaware energy-efficient resource allocation for device-to-device communications underlaying cellular networks," in Global Communications Conference (GLOBECOM), 2014 IEEE, Dec 2014, pp. 4454-4459.

[11] W. Dinkelbach, "On nonlinear fractional programming," Management Science, vol. 13, no. 7, pp. 492-498, 1967. [Online]. Available: http://www.jstor.org/stable/2627691 